\title{
Large-Scale Computational Screening of Zeolites for Ethane/Ethene Separation
}

Jihan Kim, ${ }^{* \dagger, \ddagger}$ Li-Chiang Lin, ${ }^{\S, \ddagger}$ Richard L. Martin," Joseph A. Swisher, ${ }^{\S}$ Maciej Haranczyk, $"$ and Berend Smit ${ }^{\dagger} \S$

${ }^{\dagger}$ Materials Sciences Division, Lawrence Berkeley National Laboratory, Berkeley, California 94720, United States

${ }^{\S}$ Department of Chemical and Biomolecular Engineering, University of California, Berkeley, California 94720, United States

"Computational Research Division, Lawrence Berkeley National Laboratory, Berkeley, California 94720, United States

\section{Supporting Information}

ABSTRACT: Large-scale computational screening of thirty thousand zeolite structures was conducted to find optimal structures for separation of ethane/ethene mixtures. Efficient grand canonical Monte Carlo (GCMC) simulations were performed with graphics processing units (GPUs) to obtain pure component adsorption isotherms for both ethane and ethene. We have utilized the ideal adsorbed solution theory (IAST) to obtain the mixture isotherms, which were used to evaluate the performance of each zeolite structure based on its working capacity and selectivity. In our analysis, we have determined that specific arrangements of zeolite framework atoms create sites for the preferential adsorption of ethane

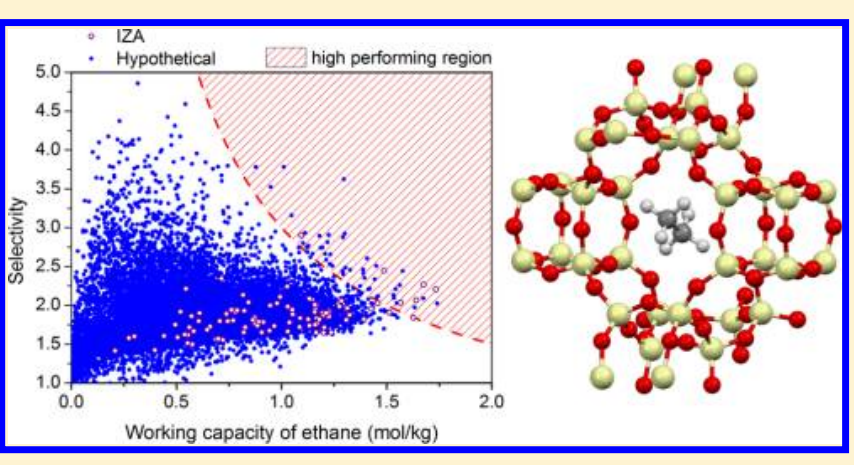
over ethene. The majority of optimum separation materials can be identified by utilizing this knowledge and screening structures for the presence of this feature will enable the efficient selection of promising candidate materials for ethane/ethene separation prior to performing molecular simulations.

\section{INTRODUCTION}

Ethene is one of the largest production chemical products today due in large part to the demand for polyethylene. Global annual production capacity exceeds 152 million tonnes and is projected to grow by $20 \%$ over the next 5 years. ${ }^{1}$ Large-scale production of ethene involves separating it from other light hydrocarbons, including methane, ethane, and propane. To achieve acceptable purity, these compounds are typically separated using a series of low-temperature distillations at high pressure. ${ }^{2}$ The low relative volatility of the ethane-ethene mixture makes the separation energy and capital intensive. Several other approaches for separating light olefins and paraffins have been proposed in the literature. These include physical and chemical absorption processes, extractive distillation, membranes, and adsorption onto porous materials. ${ }^{2-8}$

The similarity of molecular sizes in these mixtures contributes to the difficulty of the separation. Adsorbent materials can potentially provide better separation of these components. By interacting more strongly with one component of the mixture, an adsorbent may provide an adsorbed mixture richer in one component or limit the diffusion of one component for a kinetic separation. ${ }^{3}$ Previous efforts with porous materials have looked into both physically adsorbing materials, such as zeolites, ${ }^{7}$ and chemically adsorbing materials, such as metal-organic frameworks (MOFs) with open metal sites. $^{5,6}$ In addition, zeolitic imidazolate frameworks and specifically ZIF-8 has been investigated as a potential candidate for performing kinetic separation via the enhanced diffusivity of ethene compared to ethane within the material. ${ }^{4}$ For this work, we focus on zeolite structures as a possible candidate for adsorption-based separation and focus on ethane/ethene separation.

Zeolites are porous aluminosilicates with pore diameters on the order of 0.5 to $5 \mathrm{~nm}$. While roughly 200 zeolite topologies have been identified in experiments, only a handful of these materials are used on an industrial scale. Despite the relatively small number of experimentally realized zeolite structures, recent work in predicting zeolite structures has identified millions of potential pure-silica zeolites. ${ }^{9}$ Due to the very large number of these hypothetical zeolite structures that can be utilized for ethane/ethene separation, we have used computational techniques to predict the thermodynamics of both ethane and ethene molecules adsorbed on these materials and conducted detailed analysis of their crystal structures. Grand canonical Monte Carlo (GCMC) simulations were performed to obtain uptake of ethane and ethene for zeolite structures in both the IZA database and Deem's hypothetical database. ${ }^{10}$ With the development of our high performance adsorption

Received: June 1, 2012

Revised: July 6, 2012

Published: July 11, 2012 
isotherm simulation code, we have the capability to characterize a large database of porous materials within a reasonable computing time. ${ }^{11}$ Previously, we have utilized this software to conduct large-scale screening simulations to identify optimal materials for carbon capture ${ }^{12}$ and to perform in-depth analysis on promising MOF materials. ${ }^{13}$ There has been other simulation work that used different techniques to process a large database of zeolite materials. ${ }^{14,15}$ In this paper, we have extended the scope of our simulation tool to explore a large database of zeolite structures for ethane/ethene separation.

\section{METHODOLOGY}

GCMC simulations are widely used to predict the adsorption isotherms of pure and mixed gas in porous materials. At each point on the isotherm, a constant chemical potential (fugacity) of the gas, volume, and temperature are specified ${ }^{16}$ to obtain the uptake values of ethane/ethene molecules. Molecular simulations of ethane in different zeolites have been carried out by many groups ${ }^{17-22}$ while ethene has been studied far less. ${ }^{23-25} \mathrm{~A}$ united-atom force field that uses the 12-6 Lennard-Jones potential model was developed for alkanes and alkenes in the zeolites by Smit and co-workers, and it has been validated to reproduce the vapor liquid equilibrium of guest molecules. ${ }^{26,27}$ The simulated adsorption isotherms computed using this force field show excellent agreement with the experimental data for several different known zeolite structures, and thus, we assumed the force field to be transferable to all pure-silica zeolite structures.

To save computational time, a box composed of few, multiple unit cells of the material extending at least twice the distance of the cutoff radius, $R_{\text {cut }}=12 \AA$, in all directions is used for the simulation domain. All the interactions are cut off and shifted to zero at pairwise distances larger than $R_{\text {cut }}$ without any tail corrections. The zeolite framework is regarded as being rigid, and periodic boundary conditions are applied. In the GCMC simulation, several million configurations are sampled through random insertion, deletion, and translation moves of a single guest molecule in order to obtain an accurate ensemble average.

For the hypothetical zeolite database, the PCOD (predicted crystallography open database) set of 330000 structures within +30 $\mathrm{kJ} \mathrm{mol}^{-1} \mathrm{Si}$ of $\alpha$-quartz ${ }^{9}$ was further reduced down to 139397 via removal of all zeolite structures with a free sphere below $3.25 \AA$ (in which case, the pore sizes are too small, prohibiting methane to enter into the zeolite) utilizing Zeo++. ${ }^{28}$ We conducted GCMC simulations and characterized 30000 randomly chosen zeolite structures from the filtered 139397 hypothetical zeolite set in order to further reduce computational cost while still exploring a significant portion of the filtered zeolite set. Because we consider zeolite structures with the same chemistry (i.e., silicon and oxygen) and assumed the rigidity of the framework, the structure's geometry is the main factor that determines the material's separation performance. Given that small changes in the geometry lead to varying adsorption properties, a very large number of zeolite structures need to be examined in the screening process to find those suitable for ethane/ethene separation.

To perform large scale screening for ethane/ethene separation, we utilized various techniques to accelerate the GCMC simulations. ${ }^{29}$ Prior to the MC moves, energy grids with a spacing of $0.1 \AA$ are computed and stored for all interactions between the framework and the guest atoms. Density-biased sampling techniques are utilized to make biased insertion moves into low energy regions within the simulation volume to speed up the MC equilibration process. Finally, we utilize the thousands of GPU threads within the graphics processing units (GPUs) hardware to efficiently allocate threads to workload during the GCMC simulations. ${ }^{11,30}$ With all of these optimization techniques in place, it takes only a few minutes of total wall time to obtain a complete pure component isotherm up to 100 bar (fugacity of the gas).

\section{RESULTS}

Mixture isotherms are the most essential information used to evaluate the separation performance of a multicomponent system. However, direct mixture isotherm calculations can be inefficient given that the composition of a mixture input stream of process might also be a variable, and recalculations of the isotherms would be required for different ratios of the mixture. Subsequently, in the context of our large-scale screening, while the performance of a zeolite structure was still measured from the mixture isotherms, predictions were made from the ideal adsorbed solution theory (IAST) with the pure component isotherms calculations ${ }^{31}$ instead. The IAST theory is a widely used method to predict the mixture isotherm and has been validated in many adsorption systems. ${ }^{32}$

Pure Components. The pure component isotherms for both ethane and ethene molecules inside zeolite structures were obtained through GCMC simulations over a wide range of fugacities. It is interesting to note that two different types of isotherms emerged from our large-scale adsorption isotherm calculations. Figure 1 shows the pure component isotherms of

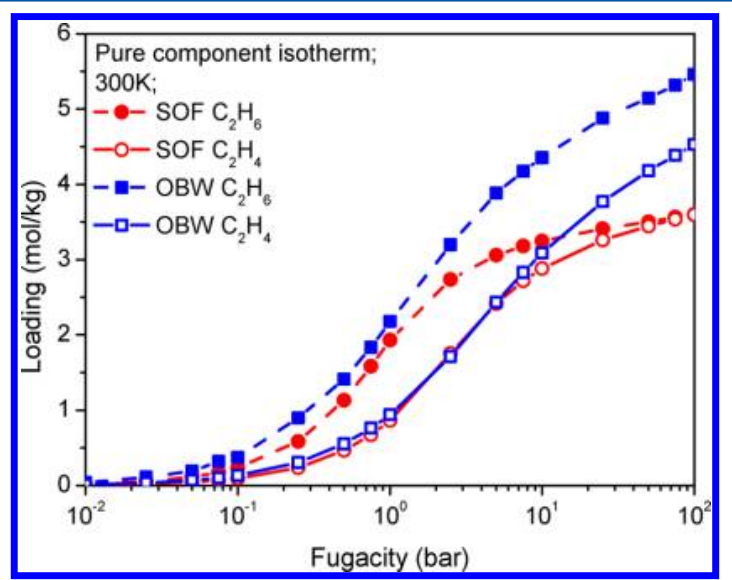

Figure 1. Ethane (dashed line) and ethene (solid line) adsorption isotherms in OBW (blue) and SOF (red) at $300 \mathrm{~K}$ from GCMC simulations.

zeolites OBW and SOF, which were chosen as IZA structures representing these two distinct classes of isotherms. Comparisons at high fugacities (i.e., between 1 and 100 bar) indicates that, for OBW, the loading difference between ethane and ethene remains relatively constant throughout this pressure range, while, in SOF, the difference becomes smaller as the uptake values of ethane and ethene converge to the same saturation point of around $3.5 \mathrm{~mol} / \mathrm{kg}$ at very high fugacities of gas. As will be shown later in the paper, the difference in saturation behavior between the pure component isotherms serves as an important indicator that determines the separation characteristics of the zeolite materials.

In order to explain the different isotherm behaviors between OBW and SOF, we utilized the GPU code to generate the energy profiles of ethane and ethene inside the two structures. Figure 2 shows the energy contour profiles, representing the sum of the potential energies between the ethane/ethene molecules and all of the framework atoms within the unit cell of OBW, with the blue regions indicating low energy adsorption sites. Because both ethane and ethene are represented as polyatomic molecules in our simulations, the average energy of the molecule at each grid point was obtained via conducting 100 center-of-mass test rotations and collecting the Boltzmannweighted average energy for all of the random rotation moves. Comparison between the ethene (Figure 2a) and the ethane (Figure 2b) energy contour plots reveals that the ethane 


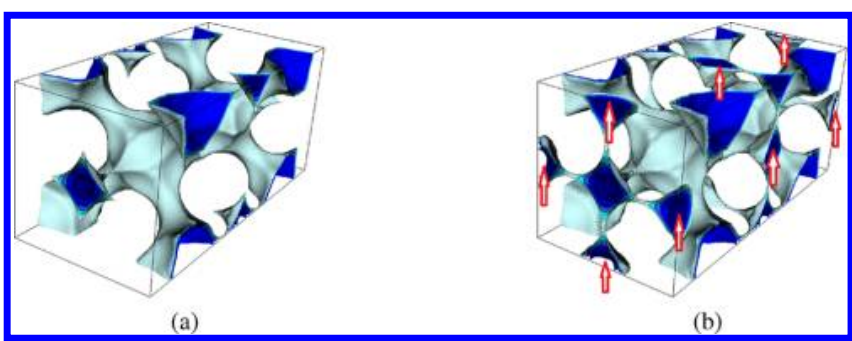

Figure 2. OBW unit cell energy contour plots for ethene (a) and ethane (b) molecules. The blue colored part indicates low energy adsorption regions within the OBW structure. The red arrows point to low energy regions that are present for ethane but absent for ethene.

molecule occupies additional regions within the unit cell that are energetically forbidden to the ethene molecule. These extra ethane adsorption sites are separated from the main channel by a relatively large energy barrier. We impose a cutoff energy value, $E_{\text {cut }}=15 k_{\mathrm{B}} T$ (with $T=300 \mathrm{~K}$ ), where an adsorption site surrounded in all spatial directions by an energy barrier higher than $E_{\text {cut }}$ is deemed to be inaccessible. In our code, we have implemented a blocking routine that allows for automatic identification of inaccessible regions via the flood fill algorithm. ${ }^{33}$ The value of $E_{\text {cut }}$ was chosen to be sufficiently large such that diffusion across this barrier is not expected within the experimental time scale. ${ }^{33}$ It turns out that, in OBW, the energy barrier separating the extra adsorption sites from the main channel is high enough to block diffusion of ethene while low enough to allow diffusion of ethane molecules as can be seen from Figure 2. Thus, changing $E_{\text {cut }}$ in our simulations can alter the diffusion properties in such a way that the adsorption properties for ethane and ethene become similar to one another. In our large-scale screening process, we have identified many zeolite structures similar to OBW, where the energy cutoff criterion imposed to enable/disable diffusion into localized regions led to extra adsorption sites for ethane. In reality, we expect the diffusion properties of ethane and ethene to be similar enough to one another in these types of zeolite structures such that the two gas molecules will either both diffuse into all of the same localized regions if the experimental time scale is long enough or be forbidden to access the same localized regions if the time scale is short enough. Although a nonequilibrium separation based on membranes, for example, can exploit these properties if the diffusion coefficients of the two molecules are sufficiently different from one another, in this study we focus on equilibrium separations and consider structures similar to OBW as being suboptimal.

On the other hand, the ethane/ethene energy contour plots for zeolite SOF (Figure 3) reveal that the low energy regions for the two molecules are same for both gas molecules. Because

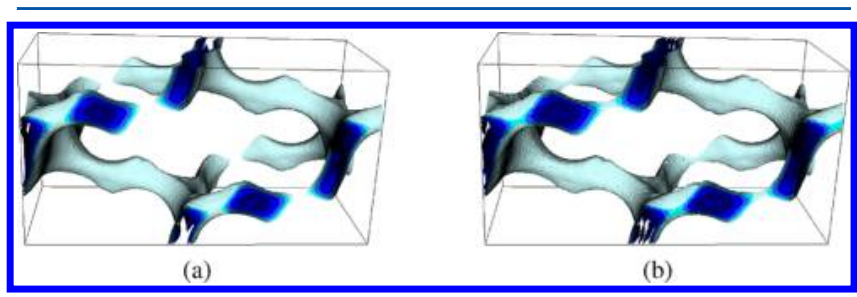

Figure 3. SOF cell energy contour plots for ethene (a) and ethane (b) molecules. Similar to Figure 2, blue indicates low energy adsorption regions, which happens to be in similar locations for both ethane and ethene in the case of SOF. ethane and ethene share the same adsorption sites, it is not surprising that the uptake of two molecules converges to the same value at high pressure as seen from Figure 1. In the rest of this paper, we concentrate on zeolite structures similar to SOF, where high working capacity and high selectivity for separation cannot be simply explained from differing diffusion behaviors (as was the case in OBW).

Mixture Isotherms. Although IAST has been validated for a variety of systems, it remains important to test its applicability in the ethene/ethane system. The SOF mixture isotherm computed using GCMC simulations was treated as a benchmark case used to determine the accuracy of IAST. The simulated adsorption isotherm curves seen in Figure 4

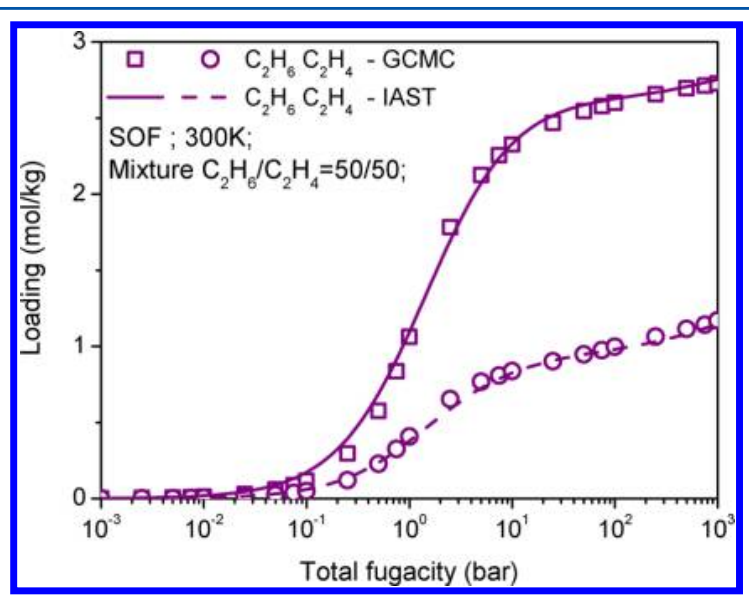

Figure 4. Open symbols show the GCMC-computed ethane-ethene (1:1) mixture isotherm in SOF at $300 \mathrm{~K}$ as a function of total fugacities. The lines show the predicted mixture isotherm by IAST.

demonstrate that the mixture isotherm data produced from pure component isotherms via IAST are in excellent agreement with the mixture isotherm data obtained from the GCMC simulations over a very wide range of fugacities.

We have also tested IAST on the zeolite OBW, and the result is shown in the Supporting Information Figure SI 1. The mixture isotherm for OBW clearly shows that IAST cannot be applied to predict the mixture isotherm accurately. The main difference between these two structures comes from the similarity of the surface area of the material accessible to each component in the mixture. As shown in Figure 2 and Figure 3, the accessible surface area of each component is nearly identical in SOF but not in OBW. More detailed information, such as identifying accessible regions for each component from molecular simulation prior to applying IAST, will improve the prediction of the mixture isotherms in materials like OBW. A detailed evaluation of IAST is out of the scope of this work and will be studied as a separate topic. In conclusion, for those structures similar to SOF studied in this paper, IAST can be used to predict the mixture behavior with high accuracy.

Screening. In order to find optimal zeolite structures that can be best utilized for ethane/ethene separation, we need to construct a criterion that separates high performance structures from the poor performing ones. In this study, we assume that a pressure swing process for an equimolar mixture of ethane/ ethene input stream was applied for the separation. Additionally, we assume that the adsorption stage is carried out at $1 \mathrm{bar}$ and $300 \mathrm{~K}$, and the desorption stage is set to be simply vacuum. Both the selectivity (i.e., $S$, ratio of ethane/ethene molecules) and ethane working capacities $(L)$ are treated as the two most 
important quantities that determine the efficiency of the ethane/ethene separation process since it would be ideal to both minimize the number of cycles needed to separate the input stream to a given purity and maximize the working capacities of ethane (i.e., loading of ethane at the adsorption stage) molecules to minimize the overall cost. Given their importance, it would seem intuitive to search for structures that maximize the product of the two aforementioned quantities (i.e., $S \times L$ ). Therefore, we chose $S \times L$ as a metric that indicates the performance of a given structure.

Figure 5 shows the two-dimensional ethane/ethene separation performance plot for 171 IZA structures as well as 30000

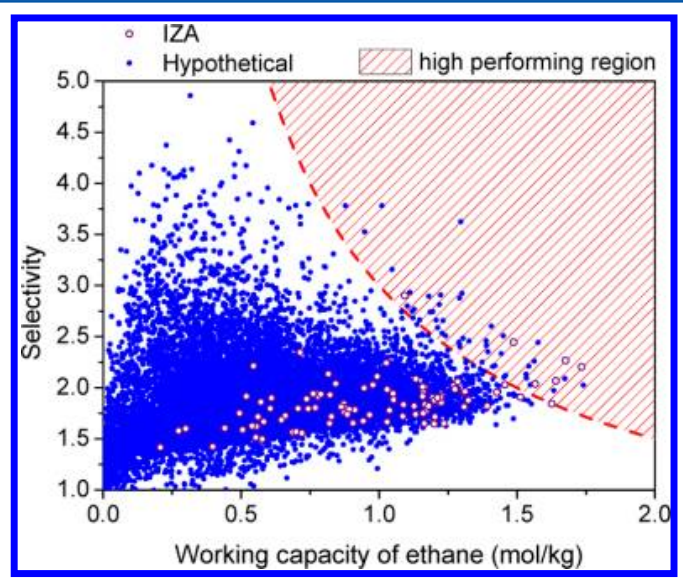

Figure 5. Two-dimensional ethane/ethene performance plot for 171 IZA structures (magenta, open circles) and 30000 hypothetical zeolite structures (blue, closed circles). For each of the points both the selectivity (vertical axis) and ethane loading (horizontal axis) are obtained from the predicted mixture isotherm by using IAST. The dashed red line represents the reference performance curve of $S \times L=$ 3 and serves to separate the high (above the line) and the low (below the line) performance zeolite structures. hypothetical zeolite structures from Deem's database. Most of the data points $(99.8 \%)$ fall below the reference performance curve of $S \times L=3$, which we utilized to separate high and low performance regions within the zeolite search space. At this point, we would like to stress that the choice of 3 here is intuitively arbitrary. The purpose of providing a reference line is to qualitatively define a number of promising materials. It is not surprising that most of the structures perform poorly given that ethane and ethene molecules are very similar to one another, resulting in similar adsorption properties for a given structure. From the top structures, we have identified that 7 out of 171 IZA structures (i.e., from the highest to lowest: zeolites EPI, STW, THO, EAB, PUN, SOF, and MEI) and 50 out of 30000 hypothetical structures lie above the $S \times L=3$ curve. The highest performing structures in the hypothetical and the IZA databases are PCOD8156587 $(S \times L=4.70)$ and zeolite EPI $(S$ $\times L=3.82)$, respectively. Included in the set of top performing structures is SOF, which possesses both high selectivity (2.9) and working capacity $(1.1 \mathrm{~mol} / \mathrm{kg})$ at the given condition of input stream in the paper. In order to explain SOF's high performance, the pairwise interactions between the gas and the host framework atoms are studied in greater detail. Figure $6 a$ shows the two energy curves that describe the Lennard-Jones interactions between $\mathrm{CH}_{2}$ of ethene and $\mathrm{CH}_{3}$ of ethane and the oxygen zeolite framework atom. In the definition of the force field, the Lennard-Jones interactions between ethane/ethene molecules and the silicon framework atoms are assumed to be zero. To obtain high selectivity and uptake of ethane molecule, there needs to be a specific localized region within the zeolite structures such that (a) the interaction between $\mathrm{CH}_{3}$ and oxygen is maximized while the $\mathrm{CH}_{2}$-oxygen interaction is suboptimal (leading to high selectivity) and (b) the number of such localized regions is maximized (leading to high uptake). In order to gain a large enough energy difference that translates into distinct, high-selective adsorption properties for the ethane/ethene molecules, the two sites within the coarsegrained guest molecule need to be surrounded by as many

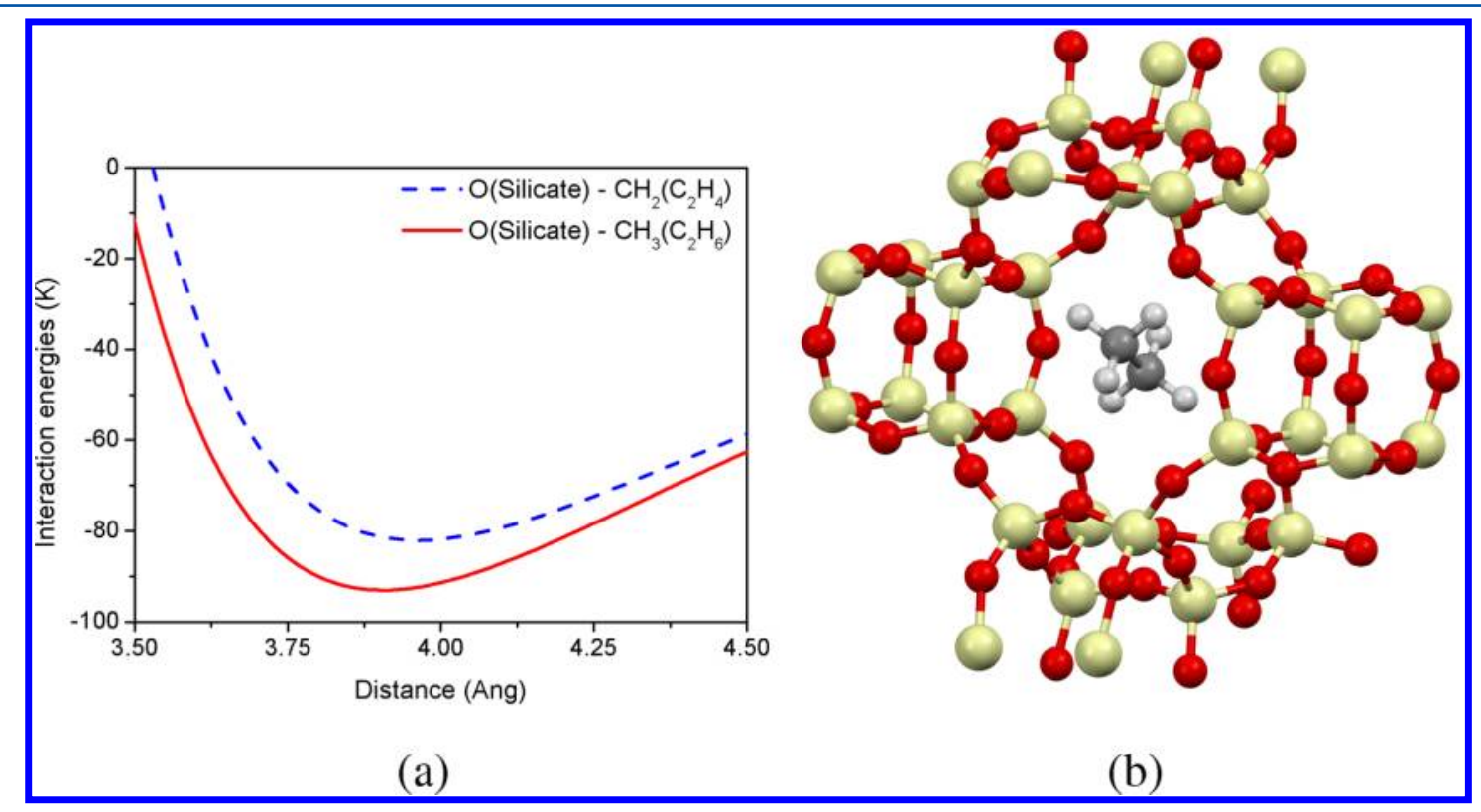

Figure 6. (a) The $\mathrm{CH}_{2}$-oxygen and $\mathrm{CH}_{3}$-oxygen Lennard-Jones interaction as a function of distance. The minimum interaction energy for $\mathrm{CH}_{2}-$ oxygen $\left(\mathrm{CH}_{3}-\right.$ oxygen) occurs at a distance of 3.96 (3.90) $\AA$. (b) A localized region of framework atoms that lead to high performance ethane/ ethene separation in SOF. 
oxygen atoms as possible in a localized region radius of roughly $3.9 \AA$ where the distance creates the largest binding energy difference between $\mathrm{CH}_{3}$-oxygen and $\mathrm{CH}_{2}$-oxygen interactions. The zeolite framework geometry depicted in Figure $6 \mathrm{~b}$ shows a channel diameter of about $7 \AA$ in the zeolite SOF that provides a localized region optimal for preferential adsorption of ethane over ethene.

Since all structures examined in this study are pure-silica zeolites and so share the same chemical composition, framework geometry, rather than chemistry, is the main factor which determines a material's separation performance. Having determined the characteristics of preferential sites for ethane adsorption in zeolite frameworks, one can utilize cheminformatics to efficiently screen a large database of zeolite materials to identify those structures that contain this specific preferential geometry without the need to conduct molecular simulations. ${ }^{34}$ Through inspection of the potential energy surface, we identified the specific zeolite framework geometry present in the preferential ethane binding site of the optimal separation material, PCOD8156587 (highest $S \times L$ metric); the arrangement of zeolite atoms around one $\mathrm{CH}_{3}$ group of the guest is shown in Figure 7, and coordinates are provided in the

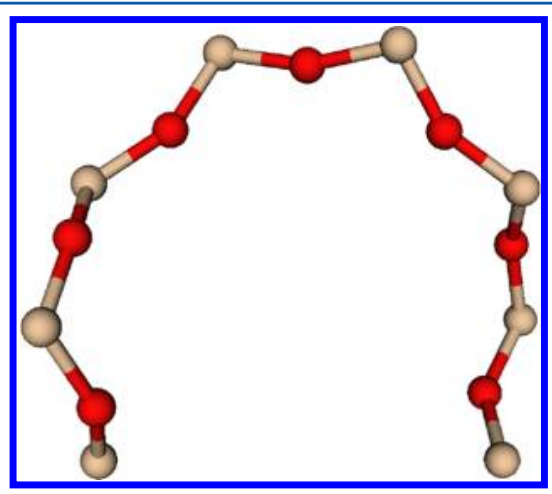

Figure 7. The illustrated arrangement of zeolite framework atoms was identified in the preferential ethane binding region of the best performing separation material, PCOD8156587. A pair of such framework atom arrangements, one around each $\mathrm{CH}_{3}$ group of a guest ethane molecule, gives rise to a large number of favorable guesthost interactions, leading to preferential ethane adsorption.

Supporting Information. Two zeolite framework atom arrangements similar to this, in close proximity in a guest-accessible channel, will give rise to strong ethane-host interaction due to the large number of framework atoms at an optimal distance from each $\mathrm{CH}_{3}$ group of the ethane molecule. Given this understanding of the optimum framework geometry, we can identify other materials which exhibit similar framework atom arrangements, following the procedure described in ref 34 . Accordingly, we find that, out of the 30 optimum separation materials with the largest $S \times L$ metric values, 27 (90\%) could be identified without the need for simulation, by searching only for this preferential framework geometry.

As a result, we have illustrated that, through high-throughput simulation, structural features that dominate adsorption performance of a material can be determined. Acccordingly, for hypothetical zeolite structures that have yet to be simulated, efficient prescreening of databases can identify promising candidate materials prior to molecular simulation, ensuring that the more computationally expensive techniques can be selectively applied. A greater degree of discrimination between promising candidate materials identified in this way is achievable through, for instance, consideration of the relative proportion of a framework's guest-accessible volume which is composed of such preferential framework geometries; however, these developments are beyond the scope of this work and will be described in a future publication.

\section{SUMMARY AND FUTURE WORK}

Utilizing a high-throughput GPU code, we have characterized adsorption properties of ethane and ethene molecules inside all of the experimentally verified IZA zeolite structures and 30000 hypothetical zeolite structures. We have observed that, due to the similarities shared between the two gas molecules, most of the zeolite structures are not suitable for ethane/ethene separation. However, we have identified a number of optimal structures, which possess both high selectivity and high ethane working capacity. Furthermore, we have determined the structural geometry that is responsible for preferential ethane adsorption and have shown that optimum ethane/ethene separation materials can be identified by screening framework geometries using this knowledge. Although the number of highperforming structures remain very small, the insights gained from our simulation results can help identify the ideal zeolite structures for ethane/ethene separation. And we believe that the procedures outlined in this paper can be extended to explore suitability of ethane/ethene separation in other classes of materials.

\section{ASSOCIATED CONTENT}

\section{S Supporting Information}

Figure depicting comparison of the IAST-predicted mixture isotherm with the GCMC-computed one in OBW and table of the Cartesian coordinates of the atoms of the preferential ethane binding geometry illustrated in Figure 7, from the framework of PCOD8156587. This material is available free of charge via the Internet at http://pubs.acs.org.

\section{AUTHOR INFORMATION}

\section{Corresponding Author}

*E-mail: jihankim@lbl.gov.

\section{Author Contributions}

† Contributed equally to this work

\section{Notes}

The authors declare no competing financial interest.

\section{ACKNOWLEDGMENTS}

J.K., R.L.M., and M.H. were supported by the Director, Office of Science, Advanced Scientific Computing Research, of the U.S. Department of Energy under Contract No. DE-AC0205CH11231. M.H. and R.L.M were supported jointly by DOE Office of Basic Energy Sciences and the Office of Advanced Scientific Computing Research through SciDAC Project \#CSNEW918 entitled "Knowledge guided screening tools for identification of porous materials for $\mathrm{CO} 2$ separations". B.S. was supported as part of the Center for Gas Separations Relevant to Clean Energy Technologies, an Energy Frontier Research Center funded by the U.S. Department of Energy, Office of Science, Office of Basic Energy Sciences under Award Number DE-SC0001015. J.A.S. was supported by the Advanced Research Projects Agency - Energy (ARPA-E), U.S. Department of Energy. L.-C.L. was supported by the Deutsche Forschungsgemeinschaft (DFG, priority program SPP 1570). This research used resources of the National Energy Research 
Scientific Computing Center, which is supported by the Office of Science of the U.S. Department of Energy under Contract No. DE-AC02-05CH11231.

\section{REFERENCES}

(1) Global Petrochemicals Overview. U.S. Petrochem. Rep. 2012, 2, $8-18$.

(2) Eldridge, R. B. Ind. Eng. Chem. Res. 1993, 32, 2208-2212.

(3) Rege, S. U.; Padin, J.; Yang, R. T. AIChE J. 1998, 44, 799-809.

(4) Bux, H.; Chmelik, C.; Krishna, R.; Caro, J. I. Membr. Sci. 2011, 369, 284-289.

(5) Bloch, E. D.; Queen, W. L.; Krishna, R.; Zadrozny, J. M.; Brown, C. M.; Long, J. R. Science 2012, 335, 1606-1610.

(6) Bao, Z.; Alnemrat, S.; Yu, L.; Vasiliev, I.; Ren, Q.; Lu, X.; Deng, S. Langmuir 2011, 27, 13554-13562.

(7) Hosseinpour, S.; Fatermi, S.; Mortazavi, Y.; Gholamhoseini, M.; Ravanchi, M. T. Sep. Sci. Technol. 2011, 46, 349-355.

(8) Shi, M.; Avila, A. M.; Yang, F.; Kuznicki, T. M.; Kuznicki, S. M. Chem. Eng. Sci. 2011, 66, 2817-2822.

(9) Pophale, R.; Deem, M. W.; Cheeseman, P. A. Phys. Chem. Chem. Phys. 2011, 13, 12407-12412.

(10) Earl, D. J.; Deem, M. W. Ind. Eng. Chem. Res. 2006, 45, 54495454

(11) Kim, J.; Martin, R. L.; Ruebel, O.; Haranczyk, M.; Smit, B. I. Chem. Theorv Comput. 2012, 8, 1684-1693.

(12) Lin, L.-C.; Berger, A.; Martin, R. L.; Kim, J.; Swisher, J. A.; Jariwala, K.; Rycroft, C. H.; Bhown, A.; Deem, M. W.; Haranczyk, M.; Smit, B. Nat. Mater. 2012, 11, 633-641.

(13) Dzubak, A.; Lin, L.-C.; Kim, J.; Swisher, J. A.; Poloni, R.; Maximoff, S.; Smit, B.; Gagliardi, L. Nat. Chem. 2012, DOI: 10.1038/ NCHEM.1432.

(14) First, E. L.; Gounaris, C. E.; Wei, J.; Floudas, C. A. Phvs. Chem. Chem. Phvs. 2011, 13, 17339-17358.

(15) Haldoupis, E.; Nair, S.; Sholl, D. S. Phys. Chem. Chem. Phvs. 2011, 13, 5053-5060.

(16) Frenkel, D.; Smit, B. Understanding Molecular Simulation: From Algorithms to Applications, 2nd ed.; Academic Press: San Diego, CA, 2002.

(17) Nowak, A. K.; Den Ouden, C. J. J.; Pickett, S. D.; Smit, B.; Cheetham, A. K.; Post, M. F. M.; Thomas, J. M. I.Phys. Chem. 1991, 95, 848-854.

(18) Gupta, V.; Nivarthi, S. S.; McCormick, A. V.; Davis, H. T. Chem. Phvs. Lett. 1995, 247, 596-600.

(19) Smit, B. I. Phvs. Chem. 1995, 99, 5597-5603.

(20) Macedonia, M. D.; Moore, D. D.; Maginn, E. I. Langmuir 2000, $16,3823-3834$.

(21) Demontis, P.; Gonzalez, J. G.; Suffritti, G. B.; Tilocca, A. I. Am. Chem. Soc. 2001, 123, 5069-5074.

(22) Krishna, R.; van Baten, J. M. Chem. Eng. Technol. 2006, 29, 1429-1437.

(23) Pascual, P.; Ungerer, P.; Tavitian, B.; Boutin, A. $\underline{\text { I.Phys. Chem. B }}$ 2004, 108, 393-398.

(24) Fan, J. F.; Wang, Q. X.; Dong, X. D.; Xiao, H. M. L. Mol. Struct. 2003, 638, 129-134.

(25) Jakobtorweihen, S.; Hansen, N.; Keil, F. I. Mol. Phvs. 2005, 103, 471-489.

(26) Dubbeldam, D.; Calero, S.; Vlugt, T. J. H.; Krishna, R.; Maesen, T. L. M.; Beerdsen, E.; Smit, B. Phys. Rev. Lett. 2004, 93 (8), 248301.

(27) Liu, B.; Smit, B.; Rey, F.; Valencia, S.; Calero, S. I. Phvs. Chem. C 2008, 112, 2492-2498.

(28) Willems, T. F.; Rycroft, C. H.; Kazi, M.; Meza, J. C.; Haranczyk, M. Microporous Mesoporous Mater. 2012, 149, 134-141.

(29) Kim, J.; Smit, B. I. Chem. Theorv Comput. 2012, 8 (7), 23362343, DOI: $10.1021 /$ ct3003699.

(30) Kim, J.; Rodgers, J.; Athènes, M.; Smit, B. I. Chem. Theory Comput. 2011, 7, 3208-3222.

(31) Myers, A. L.; Prausnitz, J. M. AIChE J. 1965, 11, 121-130.

(32) Smit, B.; Maesen, T. L. M. Chem. Rev. 2008, 108, 4125-4184.
(33) (a) Haranczyk, M.; Sethian, J. A. Proc. Natl. Acad. Sci. U. S. A. 2009, 106, 21472-21477. (b) Haranczyk, M.; Sethian, J. A. I. Chem. Theorv Comput. 2010, 6, 3472-3480. (c) Martin, R. L.; Prabhat, Mr.; Donofrio, D. D.; Sethian, J. A.; Haranczyk, M. Int. I. High Perform. C.; DOI: $10.1177 / 1094342011431591$

(34) Martin, R. L.; Willems, T. F.; Lin, L.-C.; Kim, J.; Swisher, J. A.; Smit, B; Haranczyk, M. Submitted. 